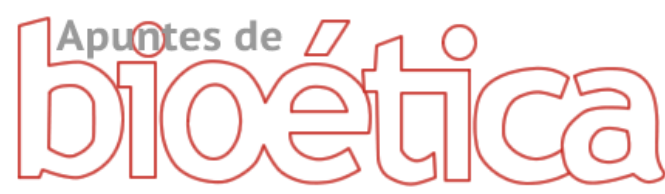

https://doi.org/10.35383/apuntes.v3i1.371

\title{
Caracterización de pacientes que reciben cuidados paliativos en una unidad privada no hospitalaria en El Salvador.
}

\author{
López-Saca M. ${ }^{1}$, Feng A. ${ }^{2}$, Carrillo F. ${ }^{3}$, Menjivar C. ${ }^{4}$ Rodríguez M. ${ }^{5}$
}

\begin{tabular}{l}
\hline INFORMACIÓN DEL ARTÍCULO \\
\hline Historia del artículo: \\
Recibido el 27 de abril 2020 \\
Aceptado el 27 de julio 2020
\end{tabular}

\section{Palabras claves:}

Cuidados Paliativos

Opioides

Síntomas

\section{RESUMEN}

En El Salvador el acceso a los cuidados paliativos es limitado. En julio de 2013 inició una unidad privada de soporte y control de síntomas en cuidados paliativos. Atienden enfermos oncológicos y no oncológicos en tres niveles: consulta externa, hospitalización en centros privados y atención domiciliaria. El objetivo de este estudio es describir las características demográficas y clínicas de la población atendida desde enero de 2014 a diciembre de 2016 como primera experiencia de atención paliativa privada en San Salvador.

Es un estudio observacional, retrospectivo y descriptivo, utilizando el censo poblacional y los expedientes clínicos. En donde se recogieron variables sociodemográficas y clínicas de los pacientes en una valoración inicial por el médico paliativista.

El total de pacientes fue 125 . Siendo $56.8 \%$ mujeres con promedio de edad 72 años. El $68 \%$ de los pacientes viven en San Salvador. $14.4 \%$ tienen seguro médico privado. $68 \%$ recibieron consulta domiciliar. El $86.4 \%$ de los pacientes atendidos padecían de enfermedades oncológicas. Los síntomas más frecuentes: debilidad $90.4 \%$, falta de apetito $88.8 \%$ y dolor $76 \%$. El opioide más utilizado fue morfina $28.8 \%$ seguido de tramadol $17.6 \%$, oxicodona $8 \%$ y fentanilo $8 \%$. Requirieron sedación paliativa $12.8 \%$. Los pacientes fueron referidos por médicos en $52.8 \%$ y por familiares $47.2 \%$.

Presentamos las características de los pacientes atendidos en la unidad privada y cuyos resultados muestran que cuando los cuidados paliativos cuidan sus principios y elementos básicos de trabajo los resultados son similares a otras unidades en el resto del mundo.

${ }^{1}$ Doctor en Medicina por la Universidad de El Salvador, Máster en Bioética de la Universidad de Navarra, Licenciado en Ciencias Humanísticas. Medicina Interna y Cuidados paliativos. Jefe de la unidad de soporte y control de síntomas. Hospital Nacional "Dr. Juan José Fernández" Zacamil, San Salvador, El Salvador. Email: mario.lopezsaca@hlacima.org. ORCID: https://orcid.org/0000-0001-6591-7918

${ }^{2}$ Doctorado en Medicina, Master en Atención Paliativa Integral a Personas con Enfermedades Avanzadas Hospital Sant Pau, Barcelona España. Medicina General y especialista en Cuidados paliativos. Médico adjunto de la unidad de soporte y control de síntomas. Universidad José Matías Delgado, San Salvador, El Salvador Email: ancu.feng@hlacima.org ORCID: https://orcid.org/0000-0002-8174-3871

${ }^{3}$ Licenciada en enfermería. Enfermera de oncología y cuidados paliativos. Enfermera coordinadora de la unidad de soporte y control de síntomas. Hospice La Cima. Centro de Medicina Paliativa, San Salvador, El Salvador Email: fcarrillo@eimed.com.sv ORCID: https://orcid.org/0000-0002-5865-3405

4 Enfermera en cuidados paliativos. Enfermera de la unidad. Hospice La Cima. Centro de Medicina Paliativa, San Salvador, El Salvador Email: cecilia.meniivar@hlacima.org ORCID: https://orcid.orq/0000-0001-8275-6335

${ }_{5}$ Psico-onocología. Psicólogo de la unidad de soporte y control de síntomas Hospital Divina Providencia, Hospicio La Cima: San Salvador, San Salvador. Email: marc.guezpaz@gmail.com ORCID: https://orcid.org/0000-0002-7119-184X 
Characterization of patients receiving palliative care in a private nonhospital unit in El Salvador

ABSTRACT

Keywords.

Palliative Care

Opioids

Symptoms
In El Salvador, access to palliative care is limited. In July 2013, he started a private support and symptom control unit in palliative care. They care for cancer and non-cancer patients at three levels: outpatient consultation, hospitalization in private centers and home care. The objective of this study is to describe the demographic and clinical characteristics of the population attended from January 2014 to December 2016 as the first experience of private palliative care in San Salvador.

It is an observational, retrospective and descriptive study, using the population census and clinical records. Where sociodemographic and clinical variables of the patients were collected in an initial assessment by the palliative doctor.

The total of patients was 125 . Being $56.8 \%$ women with an average age of 72 years. $68 \%$ of patients live in San Salvador. $14.4 \%$ have private health insurance. $68 \%$ received a home consultation. $86.4 \%$ of the patients attended suffered from oncological diseases. The most frequent symptoms: weakness $90.4 \%$, lack of appetite $88.8 \%$ and pain $76 \%$. The most widely used opioid was morphine $28.8 \%$ followed by tramadol $17.6 \%$, oxycodone $8 \%$ and fentanyl $8 \% .12 .8 \%$ required palliative sedation. The patients were referred by doctors in $52.8 \%$ and by relatives $47.2 \%$.

We present the characteristics of the patients cared for in the private unit and whose results show that when palliative care cares for its principles and basic elements of work, the results are similar to other units in the rest of the world.

\section{Introducción}

El Salvador es el país más pequeño de Centroamérica con $21,000 \mathrm{~km} 2$ pero con una alta densidad poblacional aproximadamente 6.1 millones de habitantes (CIA Library, s.f.), la esperanza de vida en el hombre es de 68 años y en la mujer de 77 años (Plan de Acción del Programa de País 2012-2015 entre El Salvador y el Fondo de Población de las Naciones Unidas. (UNFPA)). La población adulta sigue creciendo y se estima que para el año 2020 la pirámide poblacional cambie a un cono poblacional con una población mayor arriba de 60 años, esto último debido a que desde el 2014 la tasa de fecundidad es de 1.95 hijos por pareja (18Di) por debajo del 2.1 que es la tasa de recambio poblacional. Además, las cuatro principales causas de muerte identificadas en 2013 fueron: insuficiencia cardiaca, insuficiencia renal, accidentes cerebrovasculares y cáncer (Ministerio de Salud de El Salvador (MINSAL), 2014).

En el año 2014 el Ministerio de Salud de El Salvador (MINSAL) inició un programa nacional de cáncer con énfasis en la prevención que también incluyen los cuidados paliativos. Las autoridades del MINSAL inauguraron 19 unidades de dolor y cuidados paliativos en los hospitales públicos del país. Para el funcionamiento de estas unidades se crearon unos lineamientos técnicos donde además se enumera los fármacos que deberían estar disponibles para el alivio de los síntomas (MINSAL, 2014).

Otro hito para la medicina paliativa en El Salvador ha sido la ley sobre los derechos y deberes de los pacientes, promulgada en el 2016 por la Asamblea Legislativa esta incluye en el 
artículo $\mathrm{N}^{\circ} 10$ el derecho de todos los pacientes a tener acceso a los cuidados paliativos (Asamblea Legislativa de El Salvador, 2016).

Sin embargo, el acceso a los cuidados paliativos en El Salvador sigue limitado. En el Atlas Latinoamericano de Cuidados Paliativos publicado en 2012 (Pastrana, Lima, Pons y Centeno , 2013), el nivel de desarrollo de los paliativos en El Salvador es $3^{\mathrm{a}}$ (Lynch, Connor y Clark, 2013) que significa que para ese año solo contaba con una provisión aislada que es el Hospital La Divina Providencia. Otro factor que impide el desarrollo de los cuidados paliativos es la poca disponibilidad de opioides. El uso total de opioides expresado en miligramos equivalentes de morfina por habitantes es 3.5 $\mathrm{mg} / \mathrm{hab}$ estando por debajo de la media de Latinoamérica que es $4.8 \mathrm{mg} / \mathrm{hab}$ y el índice de colaboración entre prescriptores y reguladores de opioides en el país es 2, el rango del índice va de 1 a 5 , siendo este último el de máxima colaboración (Asamblea Legislativa de EI Salvador, 2016).

Un paso importante en el año 2013 fue el inicio de la primera asignatura de medicina paliativa opcional en una universidad privada para estudiantes de Doctorado en Medicina (Fortín Magaña, Goméz y López Saca, 2016). Que desde el 2018 ya es obligatoria para los alumnos de sexto año.

En el ámbito privado no existían clínicas o unidades que dieran cuidados paliativos. Hasta julio de 2013 comenzó una unidad privada de soporte y control de síntomas en cuidados paliativos. La unidad inició con un médico Paliativista formado en España, una enfermera de oncología formada en El Salvador (Centeno y Rhee, 2018). Actualmente esa unidad cuenta con 10 enfermeras, 2 médicos Paliativistas, 1 psicólogo. Atienden enfermos oncológicos y no oncológicos en tres niveles de atención: consulta externa, hospitalización en centros privados y atención en domicilio.
Esta unidad trabaja bajos principios básicos de los cuidados paliativos modernos: 1) visión global del enfermo y su familia, 2) atención de los síntomas físicos, psicológicos y existenciales, 3) además de un cambio estructural, la clínica cuenta con dos consultorios para la atención médica y psicológica, un espacio para las reuniones del equipo y un sitio para la colocación de medicamentos para el dolor y otros síntomas por la enfermera del equipo.

El objetivo de este estudio es describir las características demográficas y clínicas de la población atendida desde enero de 2014 a diciembre de 2016 ya que actualmente no existe en el país otra iniciativa como esta.

\section{Metodología}

Sujetos. Se realizó un registro total de los pacientes que acudieron al servicio de atención paliativa en una Unidad no hospitalaria de Cuidados Paliativos de tipo privada en el período enero de 2014 a diciembre de 2016, que fueron vistos tanto en la consulta como en domicilio.

Diseño. Se efectuó un estudio observacional, retrospectivo y de nivel descriptivo, utilizando un censo poblacional y los expedientes clínicos en donde se recogieron variables sociodemográficas y clínicas de los pacientes atendidos en el servicio de la Clínica de Cuidados Paliativos, recogiendo los datos de la valoración inicial del médico Paliativista.

Instrumentos. Se llevó a cabo un registro a través de una revisión de los expedientes clínicos de los pacientes en donde se recogieron las variables:

- Sociodemográficas: Edad, sexo, lugar de procedencia, atención domiciliar o consulta, si cuenta con seguro de salud.

- Clínicas: tipo de diagnóstico, Escala Karnofsky (indica la capacidad funcional del paciente), sobrevida, sedación, 
interconsulta a psicología y la escala HADS (para ansiedad y depresión), así como los síntomas más prevalentes a través de la escala de Edmonton Symptom Assessment Scale (ESAS) caracterización de delirium (a través del minimental test) y tipos de opioides.

\section{Resultados, análisis y discusión}

El total de pacientes evaluados en la Unidad de Cuidados Paliativos desde enero de 2014 hasta diciembre del 2016 fueron 125. Son del sexo femenino $n=72$ (56.8\%) con promedio de edad 72 años. Los pacientes que viven en el departamento de San Salvador son $n=85$ (68\%). Poseen seguro médico privado $\mathrm{N}=18$ (14.4\%). Consulta brindada en el domicilio n85 (68\%). Los pacientes con diagnóstico de enfermedad oncológica $n=108$ (86.4\%), 59 mujeres y 49 hombres. Los pacientes con diagnóstico de enfermedad avanzada no oncológicos $n=17$ (13.6\%), 12 mujeres y 5 hombres (Figura 1). Los pacientes referidos por médicos $n=66(52.8 \%)$, $22.4 \%$ por oncología, $24 \%$ medicina interna y cirugía, $4.8 \%$ gastroenterología y $1.6 \%$ otras especialidades. Los pacientes referidos por familiares de pacientes atendidos $n=59(47.2 \%)$. Recibieron atención por psicología $n=9$ (7.2\%), 2 mujeres y 7 hombres. (Tabla I)

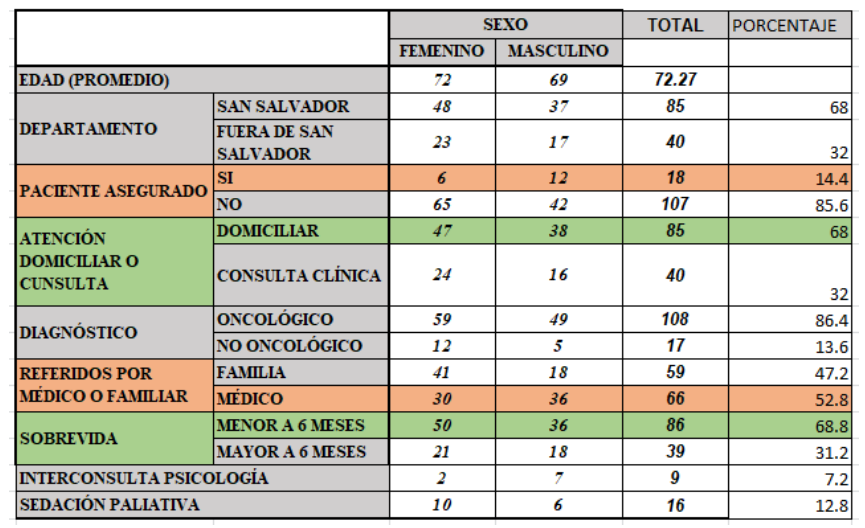

Los síntomas más frecuentes fueron: debilidad $n=113$ (90.4\%), falta de apetito $n=111(88.8 \%)$, dolor $n=95(76 \%)$, somnolencia $n=94(75.2 \%)$, insomnio $n=86(68.8 \%)$, tristeza $\mathrm{n}=78 \quad(62.4 \%), \quad$ ansiedad $\mathrm{n}=76 \quad(60.8 \%)$ estreñimiento $n=64 \quad(51.2 \%)$, náuseas $\mathrm{y} / \mathrm{o}$ vómitos $n=62(49.6 \%)$, disnea $n=47(37.6 \%)$. El delirium se presentó en $n=26$ pacientes $(20.8 \%)$, delirium hiperactivo $n=3$ (2.4\%), delirium hipoactivo $n=4(3.2 \%)$ y delirium mixto $n=19$ (15.2\%). Requirieron sedación paliativa $n=16$ (12.8\%). (Figura 1)

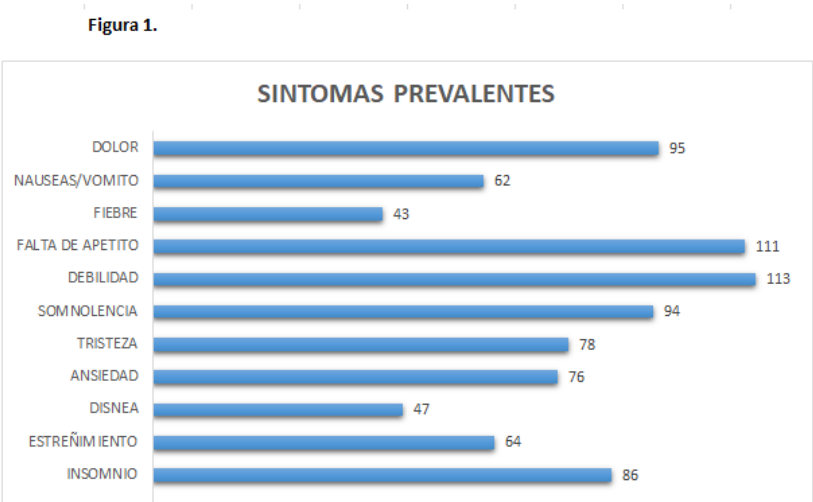

La capacidad funcional determinada por la escala de Karnofsky $\leq 50 \% \quad n=85$ (68\%), y Karnofsky $\geq 60 \% \quad n=40$ (32\%). Los pacientes con Karnofsky $\leq 50 \%$ el $77.6 \%$ son mujeres. Los pacientes con Karnofsky $\geq 60 \%$ no hubo diferencia entre el sexo. La sobrevida menor a 6 meses $\mathrm{n}=86(68.8 \%)$ y mayor a 6 meses $\mathrm{n}=39$ (31.2\%). (Figura 2)

FIGURA 2

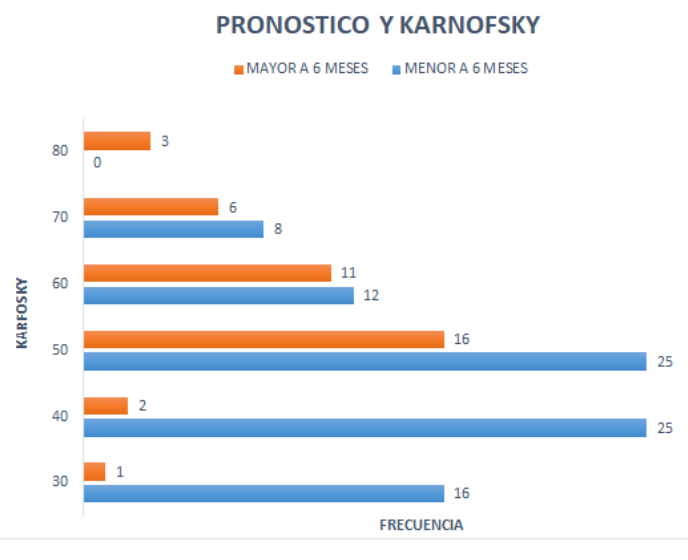

El opioide más utilizado fue morfina en $\mathrm{n}=36(28.8 \%)$ seguido por Tramadol $\mathrm{n}=22$ (17.6\%), Oxicodona $n=10(8 \%)$, Parches de 
Fentanilo $\mathrm{n}=10$ pacientes (8\%) y metadona $n=4$ (3.2\%). (Figura 3)

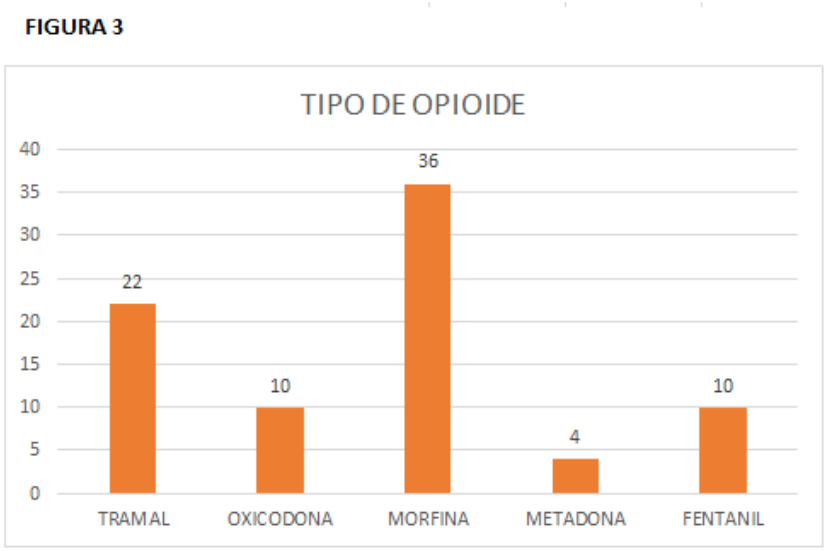

En este trabajo presentamos las características de los pacientes atendidos en nuestra unidad de cuidados paliativos. La mayoría de pacientes son mujeres ya que las principales causas de cáncer en El Salvador son cuello uterino y mama (MINSAL, 2014). La edad media de nuestros pacientes es cercana a la esperanza de vida en el país (Plan de Acción del Programa de País 2012-2015 entre El Salvador y el Fondo de Población de las Naciones Unidas. (UNFPA)).

Algunos resultados encontrados muestran la necesidad de estas unidades en el país, además de la educación y la sensibilidad en la población, por ejemplo, un buen porcentaje de pacientes cercano al $70 \%$ llegó con una capacidad funcional muy disminuida (Karnofsky $\leq 50 \%$ ) dato que refleja un tiempo de vida corto. Hay evidencia sobre la referencia tardía del paciente hacia Cuidados Paliativos (Evelia, Carlos, Silvia y Javier, 2018). Diversos estudios que demuestran que la atención temprana de los equipos de paliativos junto a las unidades de oncología de las enfermedades avanzadas provee una mejora de calidad de vida para estos pacientes sin acortar la supervivencia (Temel et al., 2010; Grudzen et al, 2010; Haun et al., 2017).
Otros datos que aportan a la sensibilización y educación de la medicina paliativa es que el diagnóstico más frecuente de pacientes vistos en la unidad fue cáncer, pocos pacientes con enfermedades no oncológicas recibieron atención a pesar de que la atención paliativa está dirigida a este tipo de enfermedades. En otros países el incremento de las intervenciones por equipos de cuidados paliativos en los pacientes con enfermedades avanzadas no oncológicas es cada vez mayor y mostrando un aumento de los cuidados paliativos domiciliares (Penders et al., 2017). Por otra parte, la referencia a la unidad por familiares de pacientes atendidos en la unidad fue muy similar a los referidos por médicos, podemos pensar que los médicos deben conocer más sobre los cuidados paliativos y que beneficio trae para sus pacientes.

En nuestra unidad se observaron la prevalencia de síntomas muy similar a otros estudios (Santiago et al., 2012; Tuca-Rodriguez et al., 2012) los principales fueron: la debilidad, hiporexia y dolor. El dolor es el síntoma más disconfortante, la mayoría de nuestros pacientes llegaron con dolor moderado y severo requiriendo la utilización de opioides de tercer escalón. El dolor en cuidados paliativos se maneja en base a la escalera analgésica propuesta por la Organización Mundial de la Salud desde los años 80 y aún sigue en vigencia, esto demuestra la eficacia de método (Gomez Sancho y Ojeda Martin).

La relación entre los prescriptores y reguladores de opioides es aún baja en el país, sin embargo, nuestra experiencia ha sido que al haber más prescripción de opioides los reguladores han facilitado el mecanismo de distribución. La creación de la Dirección Nacional de Medicamentos ha disminuido mucho la brecha entre prescriptores y reguladores, se ha creado un sistema en línea que permite al médico realizar la receta vía internet y enviarlo a la farmacia, el paciente o la familia puede comprar el medicamento solo con su documento 
de identificación. Una dificultad es que las farmacias que distribuyen los opioides solo están en la capital y no en el interior del país, tampoco están disponibles todos los opioides por ejemplo no existe aún morfina de liberación rápida solo hay morfina de liberación prolongada en una presentación $30 \mathrm{mg}$. Otras barreras es el precio alto de los opioides a nivel privado, aunque en los hospitales públicos son gratuitos. También la falta de educación en las facultades de medicina sobre uso de opioides y el manejo del dolor, sigue sumando a la opiofobia en la población y en los profesionales de la salud (Rico et al., 2015).

En otro sentido las intervenciones y abordaje de casos de los aspectos psicosociales en nuestra unidad fue bajo, el mecanismo utilizado por los médicos de nuestro equipo fue presentarlo al paciente y a la familia, cuando identificábamos que podría beneficiarse. Muchos de los equipos paliativos en otros países incluyen la figura del psicólogo dentro del equipo para soporte no solo del paciente y su familia, sino incluso para el propio equipo (Bayés y Barreto, 1993; Barreto y Bayés, 1990). No existe cultura en nuestro país que el psicólogo este dentro del equipo. Por el numero bajo de pacientes que recibieron esta ayuda pensamos que debemos buscar la estrategia adecuada para presentar al psicólogo como parte del equipo de cuidados paliativos.

La sedación paliativa es un recurso utilizado por los equipos de cuidados paliativos cuando el síntoma es refractario al tratamiento habitual (Alves et al., 2017). Aunque en los últimos años la sedación paliativa ha sido un recurso médico cada vez más utilizado, los porcentajes bajos de sedación paliativa indican buenas prácticas clínicas (Campos-Calderón et al., 2016; Chambaere et al., 2010; Schildmann, Pörnbacher et al., 2018). En un estudio reciente hecho por médicos del Hospital La Divina Providencia, único Hospice en El Salvador el 30\% de los pacientes requirieron sedación paliativa
(Ortiz y Noubleau, 2016). Nuestra unidad muestra datos más bajos (13\%).

Una de las deficiencias del trabajo es el bajo número de pacientes para tres años de atención clínica, hay que aclarar que no están incluidos los pacientes que fueron atendidos a nivel hospitalario por la dificultad de acceder a esos expedientes. Otra circunstancia que influye en el número de pacientes puede ser el hecho de ser una unidad nueva, no existe en El Salvador otra unidad con estas características.

\section{Consideraciones Éticas:}

El estudio fue retrospectivo con revisión de expedientes clínicos, no se realizó ninguna intervención en pacientes. Los expedientes solo fueron revisados por el equipo investigador (que fue el mismo equipo clínico que conocía la situación de cada paciente, no hubo otra persona ajena al equipo para extraer datos) por lo tanto la confidencialidad fue guardada, no se utilizaron nombres de pacientes.

\section{Conclusiones}

- Cuando los cuidados paliativos cuidan sus principios y elementos básicos de trabajo los resultados son similares a otras unidades en el resto del mundo.

- De acuerdo con las características observadas en nuestros pacientes, se puede concluir que la población y los profesionales de la salud detectan tardíamente la necesidad de cuidados paliativo en los enfermos avanzados. En este sentido se deben buscar mejores mecanismos de promoción, educación, sensibilización y disponibilidad de opioides en nuestro país, para mejorar la atención en los pacientes que presentan enfermedades crónicoavanzadas. 


\section{Bibliografía}

(s.f.). Recuperado el 04 de Diciembre de 2018, de

http://www.indexmundi.com/es/el_salvad or/tasa_de_fertilidad.html

Alves M, M., Abril R, R., \& Neto, I. (2017). Symptomatic Control in End-of-Life Patients. Acta Med Port. doi:10.20344/amp.7626

Asamblea Legislativa de El Salvador. (2016). Ley de Deberes y Derechos de los pacientes y prestadores de servicios de salud. Obtenido de https://rrhh.salud.gob.sv/files/webfiles/reg ulacion/ley_derecho_pacientes.pdf

Barreto, M., \& Bayés, R. (1990). El Psicólogo ante el enfermo terminal. Anales de Psicología.

Bayés, R., \& Barreto, M. (1993). Las unidades de cuidados paliativos como ejemplo de interdisciplinariedad. Clínica y Salud.

Campos-Calderón C., Montoya-Juárez C., HuesoMontoro R., Hernández-López, E., OjedaVirto, F., \& García-Caro, M. (2016). Interventions and decision-making at the end of life: the effect of establishing the terminal illness situation. BMC Palliat Care.

Centeno, C., \& Rhee, J. Y. (2018). Organization of Palliative Care in Different Parts of the World. En R. MacLeod, \& L. Van den Block (Edits.), Textbook of Palliative Care. Springer, Cham.

Chambaere, K., Bilsen, J., Cohen, J., \& et al. (2010). Continuous Deep Sedation Until Death in Belgium: A Nationwide Survey. Arch Intern Med, 490-493.

CIA Library. (s.f.). Recuperado el 04 de Diciembre de 2018, de https://www.cia.gov/library/publications/t he-world factbook/fields/2090.html
Evelia , A., Carlos, T., Silvia , A., \& Javier , P. (2018). ¿Estamos atendiendo de manera oportuna a los pacientes con enfermedad terminal? El papel de los cuidados paliativos y la nutrición. Hosp Med Clin Manag.

Fortín Magaña, M., Goméz, J., \& López Saca, M. (2016). Situación actual de la enseñanza de la medicina paliativa en las universidades de Centroamérica. Educ Med.

Gomez Sancho, M., \& Ojeda Martin, M. (s.f.). Cuidados Paliativos control de síntomas. Obtenido de http://www.mgomezsancho.com/esp/libro s/control_sintomas.pdf

Grudzen, C., Richardson, L., Johnson, P., \& et al. (2010). Emergency Department-Initiated Palliative Care in Advanced Cancer. JAMA Onco.

Haun, M., Estel, S., Rücker, G., Friederich HC, H., Villalobos, M., Thomas, M., \& Hartmann, M. (2017). Early palliative care for adults with advanced cancer. Cochrane Database Syst Rev.

Lynch, T., Connor, S., \& Clark, D. (2013). Mapping levels of palliative care development: a global update. Journal of pain and symptom management, 10941106. Obtenido de http://www.icccassociation.com/iccc53/images/stories/Articles.pdf

Ministerio de Salud de El Salvador (MINSAL). (2014). Diagnostico Situacional del Cáncer en El Salvador. Recuperado el 04 de Diciembre de 2018, de http://www.salud.gob.sv

Ministerio de Salud de El Salvador (MINSAL). (2014). Lineamientos técnicos para la atención del dolor crónico y cuidados paliativos. Recuperado el 04 de Diciembre de 2018, de http://asp.salud.gob.sv/regulacion/pdf/line amientos/lineamientos_tecnicos_atencion _dolor_cronico_y_cuidados_paliativos.pdf 
Ortiz, J., \& Noubleau, R. (2016). Indicaciones de sedación paliativa en pacientes con enfermedad oncológica avanzada ingresados en el Hospital Divina Providencia de noviembre 2014 a abril 2015. Crea Ciencia, 10(1).

Pastrana, T., Lima, L., Pons, J., \& Centeno , C. (2013). Atlas de Cuidados Paliativos de Latinoamérica. Edición cartográfica 2013.

Penders, Y., Gilissen, J., Moreels, S., Deliens, L., \& Van den Block, L. (2017). Palliative care service use by older people: Time trends from a mortality follow-back study between 2005 and 2014. Palliat Med.

Plan de Acción del Programa de País 2012-2015 entre El Salvador y el Fondo de Población de las Naciones Unidas. (UNFPA). (s.f.).

Rico, A., \& et al. (2015). Review Use of Opioids in Latin America: The Need of an EvidenceBased Change. Pain Medicine.

Santiago, A., Portela, M., Ramos, L., Larumbe, A., Urdiroz, J., Martínez, M., . . Centeno, C. (2012). A new palliative care consultation team at the oncology department of a university hospital: an assessment of initial efficiency and effectiveness. Support Care Cancer, 199-203.

Schildmann, E., Pörnbacher, S., Kalies, H., \& Bausewein, C. (2018). Palliative sedation? A retrospective cohort study on the use and labelling of continuously administered sedatives on a palliative care unit. Palliative Medicine, 32(7), 1189 $-1197$.

Temel , J., Greer, J., Muzikansky, A., Gallagher, E., Admane, S., Jackson , V., ... Lynch, T. (2010). Early palliative care for patients with metastatic non-small-cell lung cancer. N Engl J Med.

Tuca-Rodriguez, A., Gómez-Batiste, X., EspinosaRojas, J., Martínez-Muñoz, M., Codorniu, N., \& Porta-Sales, J. (2012). Structure, organization and clinical outcomes in cancer patients of hospital support teams in Spain. Supportive and Palliative Care. 